\title{
A Pragmatic Gaze on Stochastic Resonance Based Variability Tolerant Memristance Enhancement
}

\author{
Vasileios Ntinas*, Antonio Rubio \\ Department of Electronic Engineering \\ Universitat Polytécnica de Catalunya \\ Barcelona, Spain \\ \{vasileios.ntinas, antonio.rubio\}@upc.edu
}

\author{
Georgios Ch. Sirakoulis \\ ${ }^{*}$ Department of Electrical and \\ Computer Engineering \\ Democritus University of Thrace \\ Xanthi, Greece \\ \{vntinas, gsirak\}@ee.duth.gr
}

\author{
Sorin D. Cotofana \\ Department of Quantum and \\ Computer Engineering \\ Delft University of Technology \\ Delft, The Netherlands \\ S.D.Cotofana@tudelft.nl
}

\begin{abstract}
Stochastic Resonance (SR) is a nonlinear system specific phenomenon, which was demonstrated to lead to system unexpected (counterintuitive) performance improvements under certain noise conditions. Memristor, on the other hand, is a enhancement manifests as an increase of $R_{M A X} / R_{M I N}$ ratio, facilitated by noise presence, that provides higher discreteness of the ON/OFF states.

Thus, in this paper, memristor SR effects are explored, assuming various memristor models, and SR-based memristance range enhancement, tolerant to device-to- device variability, is demonstrated. Our experiments reveal that SR can induce significant $R_{M A X} / R_{M I N}$ ratio increase under up to $60 \%$ variability, getting as high as 3.4 for $29 \mathrm{dBm}$ noise power.
\end{abstract}

\section{INTRODUCTION}

The Memristor is a theoretically conceived two-terminal electrical element which total resistance varies according to the applied signal history, postulated in the seminal work of Leon Chua [1] that recently reached the spotlight of emergent nanoelectronic devices as a connection between an actual de- vice and Chua's theory [2]. After its hardware implementation, although many computing applications of memristor have been proposed [3], its most adequate current use is as memory element in Resistive Random Access Memories (ReRAM) [4]. Noteworthy, the mass production of non-volatile memristor memories has to tackle a number of problems towards the scaling of the memory capabilities. One of the most significant problems is the variability of memristor devices' charac- teristics, caused during the nanofabrication processes, that produces inconsistencies in the SET and RESET process between the devices [5], [6].

Aiming to alleviate the negative effects of memristor vari- ability, device fabrication level [7] techniques as well as error correction methods [8] have been proposed. However, device variability immunity has been also enabled by variability tolerant methods and architectures [9]-[11]. Following this direction, in this work, inspired by two different approaches from the literature, a noise-related phenomenon, known as Stochastic Resonance (SR), is exploited to enhance of the memristance range $\left(R_{M A X} / R_{M I N}\right)$ while using accurate memristor models, and its performance is demonstrated in the presence device-to-device variability. The memristance ratio, reaching 3.4 for up to $60 \%$ variability of switching rate and SET and, RESET and $29 \mathrm{dBm}$ noise power.

II. SR-Based Memristance Enhancement Stochastic Resonance (SR) is a nonlinear system specific phenomenon that exploits noise to increase the performance of

a nonlinear system for a specific range of noise power [12]- [14], extensively noticed in a wide range of systems, from biological systems to electronics [15]-[18].

Having in mind that memristor is naturally a nonlinear element, SR can effectively affect it. Thus, SR as a way to improve the performance of memristive systems has been proposed in previous works with two different approaches. The first one utilises a full spectrum noisy signal [19], known as White or Gaussian noise, while the second, in a more circuit- level effective way, introduces single-frequency noisy signal [20]. Either approach presents advantages and disadvantages, as the white noise approach proposes a more power-efficient method but less efficient to control the power of noise in circuit-level, whilst the other provides an easy way for circuit designers but the signal-to-noise ratio (SNR) of the input signal is not sufficiently high $(<1)$ for actual power-efficient applications.

\section{A. Single-Frequency Noise}

Starting from the single-frequency approach, Tanaka et al. [20] proposed a circuit configuration of two in-series sinusoidal voltage sources, one for the driving signal $\left(V_{0} \sin \left(\omega_{0} t\right)\right)$ and one for the high-frequency signal of noise $\left(V_{e} \sin \left(\omega_{e} t+\right.\right.$ $\left.\left.\varphi_{e}\right)=p V_{0} \sin \left(q \omega_{0} t+\varphi_{e}\right)\right)($ Fig. 1(a)). In this approach, the driving signal is characterised by a low-amplitude and lowfrequency sinusoidal voltage, while noise signal is more than 10 times larger in both amplitude and frequency. Fig. 1(b) presents time evolution of memristance, normalised by mini- mum value $\left(M / R_{O N}\right)$, when only the driving signal is applied on the memristor (bottom), and when the combination of driving and noise signals is applied (top). The latter exhibits a wider range of memristance values always controlled by the driving signal's phase. The optimal conditions for the 


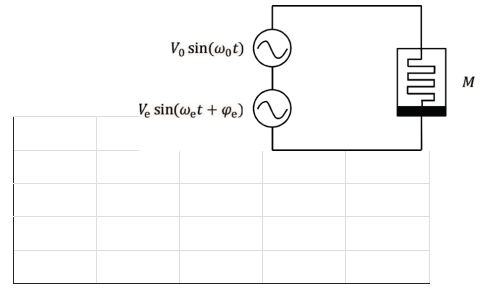

(a)

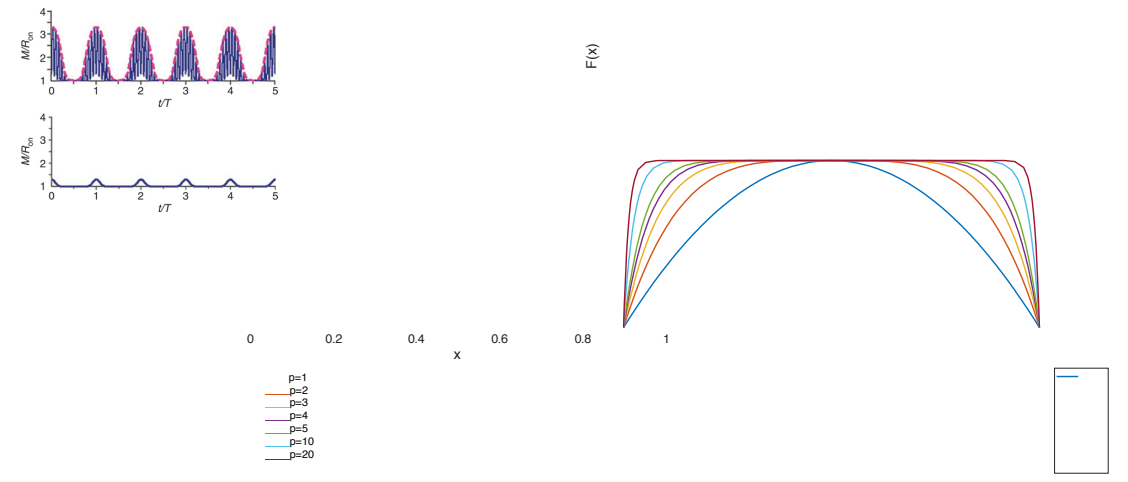

(b)

Fig. 1. (a) Tanaka's et al. [20] proposed circuit configuration and (b) results for memory enhancement (top) with and (bottom) without high-frequency input signal. (Adopted from [20])

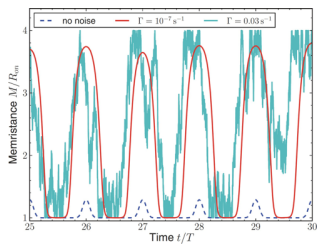

(a)

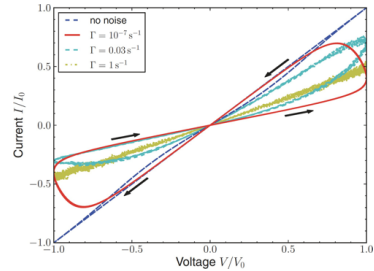

(b)

Fig. 2. (a) Memristance time evolution and (b) pinched hysteresis loop of $\mathrm{HP}$ 's $T \mathrm{iO}_{2}$ memristor linear drift model [2] under small input signal and various noise scenarios, as presented in [19]. (Adopted from [19]) achievement of the maximum memristance enhancement using this configuration are further studied in [21].

\section{B. White Noise}

Following the basic principles of SR, Stotland and DiVentra presented how the introduction of a white noise signal to the driving input signal can affect the memristance value range in a positive way [19]. Utilising the nonlinear dynamics of the memristor, the introduced noise is able to improve the quality of memristor's hysteresis loop by achieving a higher $R_{M A X} / R_{M I N}$ ratio under the same driving input signal. In Fig. 2(a), three examples with different values of noise power are presented, illustrating that for a specific amount of noise power the $R_{M A X} / R_{M I N}$ ratio can be improved. However, after a certain point, the inserted noise can result to the degradation of system's performance as the $R_{M A X} / R_{M I N}$ ratio is mostly related to the noise signal. The aforementioned effect of noise is further presented in I-V domain by Fig. 2(b).

\section{Understanding SR on linear HP memristor model}

In both of the above approaches, memristor's behaviour is described by the original HP's $\mathrm{TiO}_{2}$ memristor model [2], which maps the total memristor's resistance (Memristance, $M$ ) to two in-series variable resistors corresponding to the resistance of the undoped $\left(R_{O F} F\right)$ and the doped $\left(R_{O N}\right)$ areas of the device, with $R_{O N}<<R_{O F F}$. The total resistance of the device is calculated based on the width of each area, according to the following expression:

Fig. 3. Joglekar's window function [22] for various values of parameter $P$.

where $\mu_{V}$ is the average ion mobility. Given that the changing rate of memristance is governed by a linear expression, the requested nonlinearity for the emergence of SR-related behavior is missing.

Towards the deeper investigation of how memristance en- hancement is achieved and of its relationship with SR, the above missing point is further explored. Specifically, although Eq. (2) is a linear expression, memristor is a physical system and its state values have to be bounded. So, during the use of memristor model in [19], [20], the handling of the boundary conditions was achieved by applying the Joglekar's window function $F_{\text {window }}(x, P)=1\left(\begin{array}{lll}2 x & 1\end{array}\right)^{2 P}$ [22] (Fig. $3)$. In specific, they are multiplying Eq. (2) by the window function $F_{\text {window }}\left(\frac{w(t)}{1}, 1\right)$, which fences memristor's state $(0$ $w(t) / D$ 1) by suppressing, in nonlinear manner, its changing rate when close to the boundaries, even makes it zero when exactly at a boundary value independent of the applied signal.

The above realisation provided a useful tool on the deeper understanding of how memristance enhancement was achieved before. More particularly, setting the initial memristor state near the boundaries, the driving signal alone is incapable of changing the state sufficiently away from the boundary and as a result it hardly affects the memristance. On the other hand, the inserted noise supports the driving signal on moving the state awa from the boundaries and, therefore, the window function is lesser suppressing the state's changing rate, giving the driving signal the capability to achieve higher $R_{M A X} / R_{M I N}$ ratio. 
Delving deeper into the single-frequency memristance en- hancement approach, after exploring the mechanisms of Tanaka's et al. examples, the applicability of this approach in a wider range of memristor models is examined. Although HP's original $\mathrm{TiO}_{2}$ memristor model is the most widely used it constitutes the first attempt to model the behaviour of the actual device, while, since now, other memristor models have been proposed.

[23] in HP Labs, and the Tungsten Oxide ( $\left.W O_{x}\right)$, proposed by Chang et al. [24], memristor models have been adopted where $w(t)$ is the width of the doped area and $D$ the thickness of the thin semiconductor film. In this model, the changing rate of $w(t)$ depends on the current $i(t)$ that passes through the device and constitutes the state equation of system by the following linear expression: to explore the validity of single-frequency memristance en- hancement approach with more updated realistic models.

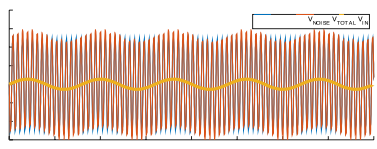

(a)

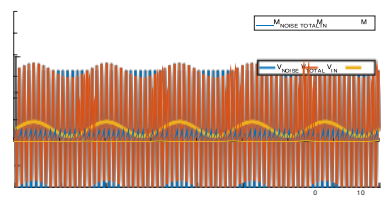

0

$\begin{array}{llll}-2 & & 1 & 0.5\end{array}$

1.80
1.75
1.70
1.65
1.60
1.55
1.50
1.45
1.40

C. 4

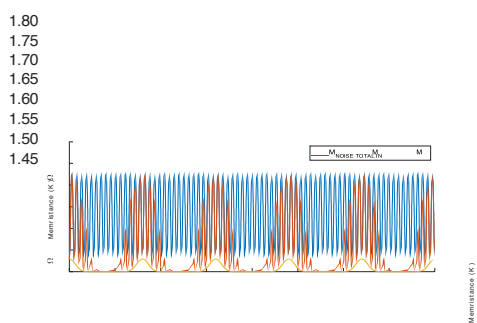

(b)

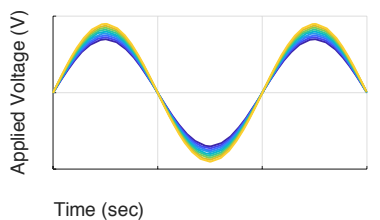

(a)

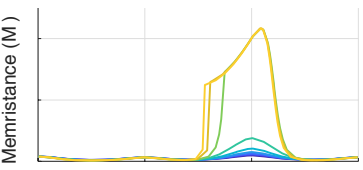

Fig. 5. $W O_{x}$ memristor model's response to sinusoidal applied voltage signals with different amplitudes $V_{p e a k}$ 
(d)
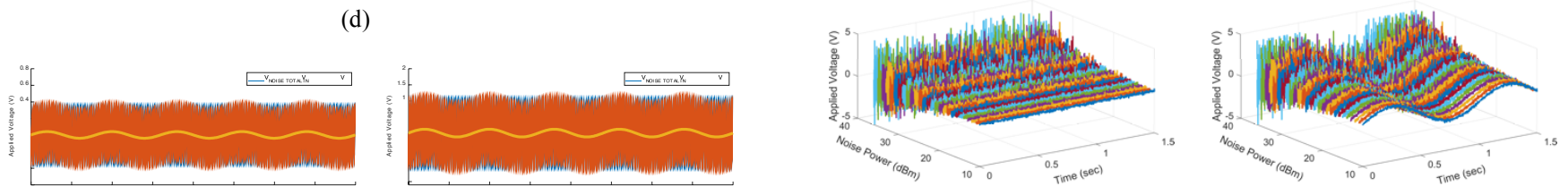

(e)

(f)

(a)

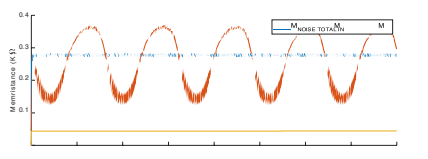

(b)

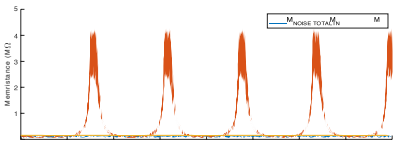

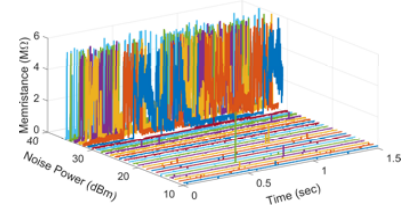

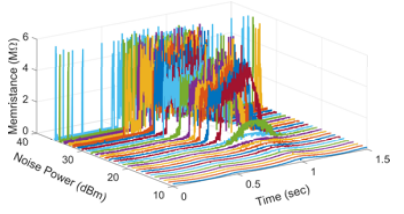


(h)

Fig. 4. (a), (b), (e), (f) Applied voltages for driving (yellow), noise (blue) and combined (orange) signals. (c), (d), (g), (h) Memristance time evolution according to the corresponding applied signals (a), (b), (e), (f), using Tanaka's example [20], modified example with $T \mathrm{iO}_{2}$ model, example with $T$ aO $\mathrm{O}_{x}$ model and example with $W \mathrm{O}_{x}$ model, respectively.

model is considered a stable and accurate memristor model that provides a robust SPICE-compatible netlist.

In specific, in order to achieve memristance enhancement with different memristor models, the applied voltage has to be modified according to each model's requirements and constrains. Thus, firstly, the original example of [20] was reproduced and in Figs. 4(a), (c), the applied signal and its effect to the memristance for the cases of only noise, driving and combined signals are illustrated. Moreover, using the knowledge acquired previously, during the study of SR on HP's model, the initial state of memristor is crucial to the emergence of memristance enhancement, so a second example with $\mathrm{TiO}_{2}$ model for initial conditions $\left(x_{0}=\underline{w_{0}}=0.9997 M_{0} \quad 1.001 K \Omega\right)$ close to the boundary value $x=1$, corresponding to $R_{O N}$ state, is further presented in Figs 4(b), (d). As result of our modification, the effect of noise alone on the memristance is negligible compared to the previous example; however, the combination of driving and noise signal is able to achieve memristance enhancement. Additionally, for the modified example, the amplitude ratio between the driving signal and the noise $(p)$ is decreased from

(c)

(d)

Fig. 6. (a) Only-noise and (b) combined signals for various noise powerlevels. (c), (d) $W O_{x}$ memristor model's response to the applied voltages signals of (a) and (b), respectivery.

the noise signal is increased radically to reduce its effect on the memristance, so from $q_{T \text { anaka }}=\underline{\omega_{e}}=15$ it is here $q=$ 200. Furthermore, in each case the value of amplitude ratio was fixed at $p=10$. Nevertheless, the amplitude of driving signal is adjusted according to model's characteristics, so $V_{0}=40 \mathrm{mV}$ and $V_{0}=120 \mathrm{mV}$ for $T a O_{x}$ and $W O_{x}$ examples, respectively.

\section{White Noise AND VARIABILITY}

Aiming to study the use of white noise on memristance enhancement for future application on actual devices, for the rest of the paper only the $W O_{x}$ memristor model is taken under consideration, as provides realistic results without the convergence issues that emerge during the simulations of $\operatorname{Ta} O_{x}$ model under the highly fluctuating voltages of the input signal.

ing signal can aid at the switching of memristor's state accord- ing to the latter signal, corresponding to higher $R_{M A X} / R_{M I N}$ ratio. So, in Figs. 6(a), (c), the effect of noise alone on a $W O_{x}$ memristor is presented for a wide range of levels of noise's power, while in Figs. 6(b), (d), the aforementioned noise signals are combined with a specific driving signal $\left(V_{\text {peak }}=1.45 \mathrm{~V}\right)$.
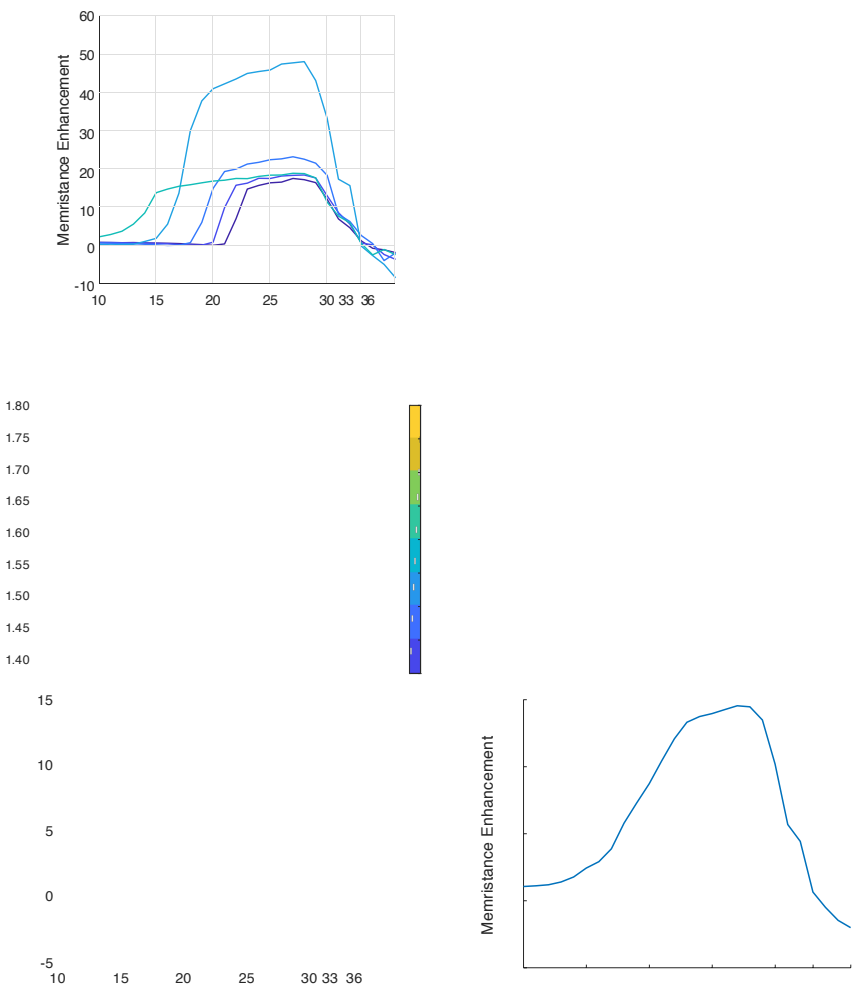

Noise Power (dBm)

(b)

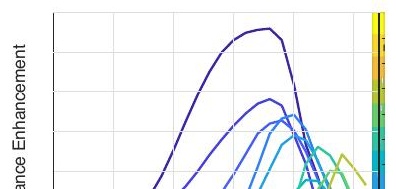



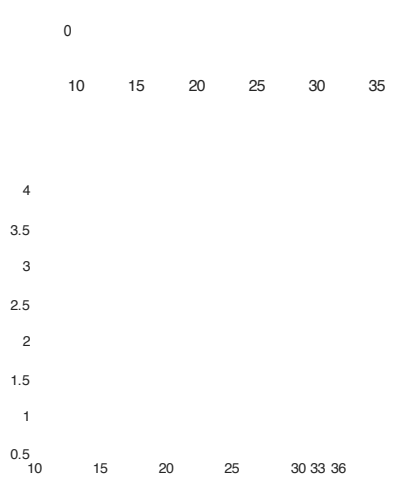

Noise Power (dBm)

(a)

(b)

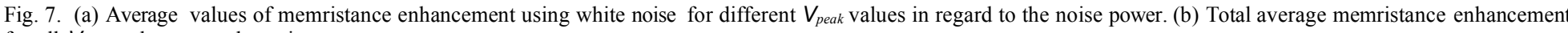
for all $V_{\text {peak }}$ values over the noise power.

Different from previous work on $\mathrm{Ti}_{x}$ model which reveal a memristance response following the input signal sinusoidal behavior [19], the dynamics of $W O_{x}$ model provides a more complicated memristance vs applied signals evolution. Thus, the memeristance enhancement evaluation in this case cannot be simply calculated as the on-resonant frequency response of combined input and noise signal over the corresponding only- noise value. So, in a way to evaluate the effect of noise on memristor's switching for the $W O_{x}$ model, the area of the memristance $(A)$ in the Memristance-Time graph was calcu- lated, as a way to measure the duration of memristance being in $R_{M A X}$ state, for each of the aforementioned cases, and the following expression is utilised to evaluate the memristance enhancement:

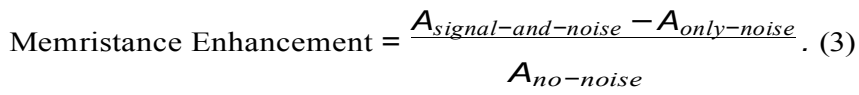

The main idea of the above calculation is to show how much the memristance range improved over the corresponding no- noise case, but also subtracting the effect of the noise alone. The average results over 50 simulations of each $V_{\text {peak }}$ value and noise power level are presented in Fig. 7(a), where is shown that increasing the $V_{\text {peak }}$ values, the enhancement is lower as driving signal achieves higher memristance value alone, even the switching of the device and the enhancement by noise is disappearing $\left(V_{\text {peak }}>1.65 \mathrm{~V}\right)$. Additionally, the general average memristance enhancement of all simulations over the noise power in Fig. 7(b) depicts the SR-like region that noise utterly improves the memristance range.

\section{Variability addressed by Memristance Enhancement}

In spite of the fact that memristance enhancement can effectively increase the range of memristance values, the exploration of its suitability on the handling of memristors' variability is evident. Given that individual memristor's be- havior inside a memristor crossbar array is varying due to the nanofabrication processes, a way to tackle with the diversion of memristor switching is necessary. Thus, at this point we performed a study on the effect of noise on memristance for a wide range of memristor devices with variability character- istics introduced on model's state equation, as shown in the following modified form of [24]'s expression:

$$
\left.\left.\frac{d w}{d t}=\left(\lambda+\delta_{\lambda}\right)\left[\exp \left(\eta_{1}+\delta_{\eta_{1}}\right) V\right)-\exp \left(\eta_{2}+\delta_{\eta_{2}}\right) V\right)\right]
$$

Fig. 8. (a) Average value of memristance enhancement using white noise for different $V$ ar values in regard to the noise power. (b) Total average memristance enhancement for all $V$ ar values over the noise power.

where $\lambda, \eta_{1}$, and $\eta_{2}$ are fitting parameters of the original model [24], corresponding to the changing rate, the $S E T$ and the $R E S E T$ threshold, respectively. So, $\delta_{\lambda}, \delta_{\eta_{1}}$, and $\delta_{\eta_{2}}$ are randomly selected fluctuations of the corresponding entities.

During our variability tests, 30 different sets of parameters $\delta_{\lambda_{2}}, \delta_{\eta_{1}}, \delta_{\eta_{2}}$ were selected by using Gaussian distribution around variability $\operatorname{Var}=5,10, \ldots, 60 \%$ of the original values of $\lambda, \eta_{1}, \lambda_{2}$ and tested for the cases of: (a) only driving signal, (b) only noise, and (c) the combination of them, for 10 to $36 \mathrm{dBm}$ noise power.

The memristance enhancement results for the aforemen- tioned simulations are deppicted in Fig. 8. In specific, the average results for each noise power level per variability percentage $V$ ar are presented in Fig. 8(a), which indicates that the memristance enhancement is diminishing (and requires higher noise levels) for high variability sets. The general average memristance enhancement of all simulations over the noise power is illustrated in Fig. 8(b), with a maximum average enhancement of $3.4 \times$ for $29 \mathrm{dBm}$ noise power.

\section{CONCLUSIONS}

In this paper, the study of the positive effect of noise, that accompanies a driving voltage applied to a memristor, is performed. Initially, a single-frequency approach of mem- ristance enhancement was adopted and simulations assum- ing accurate $\mathrm{TaO}_{x}$ and $\mathrm{WO}_{x}$ memristor models have been held, demonstrating the potential applicability of this circuit- friendly approach on actual devices. Furthermore, memristance enhancement based on white noise experiments have carried on using the robust $W O_{x}$ model in the presence ofdevice- to-device variability. Our experiments indicated that white noise based SR meristance enhancement requires higher noise levels for high device variability but it can deliver up to $3.4 R_{M A X} / R_{M I N}$ ratio improvement in the presence of up to $60 \%$ device variability, which makes it a strong candidate for practical 
implementations. As future work, the effect of noise against variability issues in resistive memory cells will be studied, aiming to the enhancement of the margin between the resistive states.

ACKNOWLEDGMENT

This work was supported in part by the Spanish MINECO and ERDF grant (TEC2016-75151-C3-2-R) and the GreeceRussia bilateral joint research project MEM-Q (proj.no./MIS T4 $\triangle \mathrm{P} \Omega-00030 / 5021467$ ) supported by GSRT and funded by National and European funds.

\section{REFERENCES}

[1] L. Chua, "Memristor-the missing circuit element," IEEE Transactions on Circuit Theory, vol. 18, no. 5, pp. 507-519, 1971.

[2] D. B. Strukov, G. S. Snider, D. R. Stewart, and R. S. Williams, "The missing memristor found," Nature, vol. 453, no. 7191, p. 80, 2008.

[3] I. Vourkas and G. C. Sirakoulis, Memristor-based nanoelectronic com-puting circuits and architectures. Springer, 2016.

[4] H. Akinaga and H. Shima, "Resistive random access memory (ReRAM) based on metal oxides," Proceedings of the IEEE, vol. 98, no. 12, pp. 2237-2251, 2010.

[5] A. Fantini, L. Goux, R. Degraeve, D. Wouters, N. Raghavan, G. Kar, A. Belmonte, Y.-Y. Chen, B. Govoreanu, and M. Jurczak, "Intrinsic switching variability in HfO2 RRAM," in Memory Workshop (IMW), 2013 5th IEEE International. IEEE, 2013, pp. 30-33.

[6] S. Ambrogio, S. Balatti, V. McCaffrey, D. C. Wang, and D. Ielmini, "Noise-induced resistance broadening in resistive switching memory Part I: intrinsic cell behavior," IEEE Transactions on Electron Devices, vol. 62, no. 11, pp. 3805-3811, 2015.

[7] J. Park, S. Jung, W. Lee, S. Kim, J. Shin, D. Lee, J. Woo, and H. Hwang, "Improved switching variability and stability by activating a single conductive filament," IEEE Electron Device Letters, vol. 33, no. 5, pp. 646-648, 2012.

[8] S. Ning, "Advanced Bit Flip Concatenates BCH Code Demonstrates 0.93\% Correctable BER and Faster Decoding on (36,864, 32,768) Emerging Memories," IEEE Transactions on Circuits and Systems I: Regular Papers, no. 99, pp. 1-9, 2018.

[9] D. Querlioz, O. Bichler, and C. Gamrat, "Simulation of a memristor- based spiking neural network immune to device variations," in Neural Networks (IJCNN), The 2011 International Joint Conference on. IEEE, 2011, pp. 1775-1781.

[10] D. Chabi, W. Zhao, D. Querlioz, and J.-O. Klein, "Robust neural logic block (nlb) based on memristor crossbar array," in Nanoscale Architectures (NANOARCH), 2011 IEEE/ACM International Symposium on. IEEE, 2011, pp. 137-143.

[11] F. Alibart, L. Gao, B. D. Hoskins, and D. B. Strukov, "High precision tuning of state for memristive devices by adaptable variation-tolerant algorithm," Nanotechnology, vol. 23, no. 7, p. $075201,2012$.

[12] R. Benzi, A. Sutera, and A. Vulpiani, “The mechanism of stochastic resonance," Journal of Physics A: mathematical and general, vol. 14, no. 11, p. L453, 1981.

[13] B. McNamara and K. Wiesenfeld, "Theory of stochastic resonance," Physical Review A, vol. 39, no. 9, p. 4854, 1989.

[14] M. D. McDonnell and D. Abbott, "What is stochastic resonance? Definitions, Misconceptions, Debates, and its relevance to biology," PLoS computational biology, vol. 5, no. 5, p. e1000348, 2009.

[15] K. Wiesenfeld and F. Moss, "Stochastic resonance and the benefits of noise: from ice ages to crayfish and squids," Nature, vol. 373 , no. 6509, p. $33,1995$.

[16] L. Gammaitoni, P. Hänggi, P. Jung, and F. Marchesoni, "Stochastic resonance," Reviews of modern physics, vol. 70, no. 1, p. $223,1998$.

[17] R. Mantegna and B. Spagnolo, "Stochastic resonance in a tunnel diode," Physical Review E, vol. 49, no. 3, p. R1792, 1994.

[18] S. Fauve and F. Heslot, "Stochastic resonance in a bistable system," Physics Letters A, vol. 97, no. 1-2, pp. 5-7, 1983

[19] A. Stotland and M. Di Ventra, "Stochastic memory: memory enhance- ment due to noise," Physical Review E, vol. 85, no. 1, p. $011116,2012$.

[20] H. Tanaka, Y.Tadokoro, and H. Iizuka, "Memristance enhancement by external voltage source," Electronics Letters, vol. 49, no. 23, pp. 1446- 1448, 2013.

[21] H. Tanaka, Y. Tadokoro, and H. Iizuka, "Optimal condition of memris- tance enhancement circuit using external voltage source," AIP Advances, vol. 4, no. 5, p. 057117, 2014.

[22] Y.N. Joglekar and S. J. Wolf, “The elusive memristor: properties of basic electrical circuits," European Journal of Physics, vol. 30, no. 4, p. 661, 2009.

[23] J. P. Strachan, A. C. Torrezan, F. Miao, M. D. Pickett, J. J. Yang, W. Yi, G. Medeiros-Ribeiro, and R. S. Williams, "State dynamics and modeling of tantalum oxide memristors," IEEE Transactions on Electron Devices, vol. 60, no. 7, pp. 2194-2202, 2013.

[24] T. Chang, S.-H. Jo, K.-H. Kim, P. Sheridan, S. Gaba, and W. Lu, "Synaptic behaviors and modeling of a metal oxide memristive device," Applied physics A, vol. 102, no. 4 , pp. 857-863, 2011 
[25] V. Ntinas, A. Ascoli, R. Tetzlaff, and G. C. Sirakoulis, "A complete analytical solution for the on and off dynamic equations of a TaO memristor," IEEE Transactions on Circuits and Systems II: Express Briefs, 2018. 